Cinémas

Revue d'études cinématographiques

Journal of Film Studies

\title{
La mort de Dante
}

\section{Jacques Aumont}

Volume 8, numéro 1-2, automne 1997

Cinéma et mélancolie

URI : https://id.erudit.org/iderudit/024746ar

DOI : https://doi.org/10.7202/024746ar

Aller au sommaire du numéro

Éditeur(s)

Cinémas

ISSN

1181-6945 (imprimé)

1705-6500 (numérique)

Découvrir la revue

Citer cet article

Aumont, J. (1997). La mort de Dante. Cinémas, 8(1-2), 125-145.

https://doi.org/10.7202/024746ar

\section{Résumé de l'article}

L'histoire que pratique Jean-Luc Godard dans ses Histoire(s) du cinéma (1986-1997) est une histoire philosophique où la Raison est synonyme de chaos, de perte et d'éloignement de l'Esprit. Au fil des épisodes, la perte devient celle de l'enfance de l'art cinématographique. L'histoire du cinéma apparaît, à l'historien philosophe et cinéaste, comme l'histoire d'une perte de l'Art, mais d'une perte paradoxale, puisqu'elle se décrit aussi comme l'histoire d'une « fin » à l'Art. d'utilisation que vous pouvez consulter en ligne.

https://apropos.erudit.org/fr/usagers/politique-dutilisation/ 


\section{La mort de Dante}

\section{Jacques Aumont}

\section{RÉSUMÉ}

Lhistoire que pratique Jean-Luc Godard dans ses Histoire(s) du cinéma (1986-1997) est une histoire philosophique ciù la Raison est synonyme de chaos, de perte et d'eloignement de l'Esprit. Au fil des épisodes, la perte devient celle de l'enfance de l'art cinématographique. Lhistoire du cinéma apparaît, à l'historien philosophe et cinéaste, comme l'histoire d'une perte de l'Art, mais d'une perte paradoxale, puisqu'elle se décrit aussi comme l'histoire d'une "fin " à l'Art.

\section{ABSTRACT}

This history practiced by Jean-Luc Godard in his Histoire(s) du cinema (1986-1997) is a philosophical history in which Reason is synonymous with chaos, loss and the distancing of the spirit. Through the episodes, the loss portrayed is that of the infancy of cinematographic art. Film history appears, for this philosophical and film-making historian, as a history of the loss of Art, a paradoxical loss, since it describes itself also as the history of an "end" to Art.

Cura enim quia prima finxit, teneat quamdiu vixerit.

Hyginus

La mia alegrez' e la maninconia

$\mathrm{E}$ « I mio riposo son " questi disagi.

Michel-Ange 
Jean-Luc Godard commence, vers 1986, en même temps qu'il réalise King Lear et Soigne ta droite, une série d'essais filmés intitulés dès le début Histoire(s) du cinéma. Il y reprend le principe et la préoccupation qui avaient été siens dans une série de conférences données en 1976 à Montréal, et publiées en 1980 sous le titre d'Introduction à une véritable histoire du cinéma.

Les conférences canadiennes avaient adopté un principe unique, celui de la confrontation, à chaque fois, entre un film de Godard et de longs extraits de quelques autres films qui avaient un rapport avec son film. Juste en même temps ou juste après, les deux séries télévisuelles Six fois deux, sur et sous la communication et France Tour Détour Deux Enfants (1980), adoptaient elles aussi un modèle unique: 12 épisodes groupés en six fois deux, chacun respectant un découpage, une "grille" a priori. Les Histoire(s) ont d'abord été imaginées pour combiner ces deux principes, de la confrontation des films et de la structure épisodique; l'ensemble

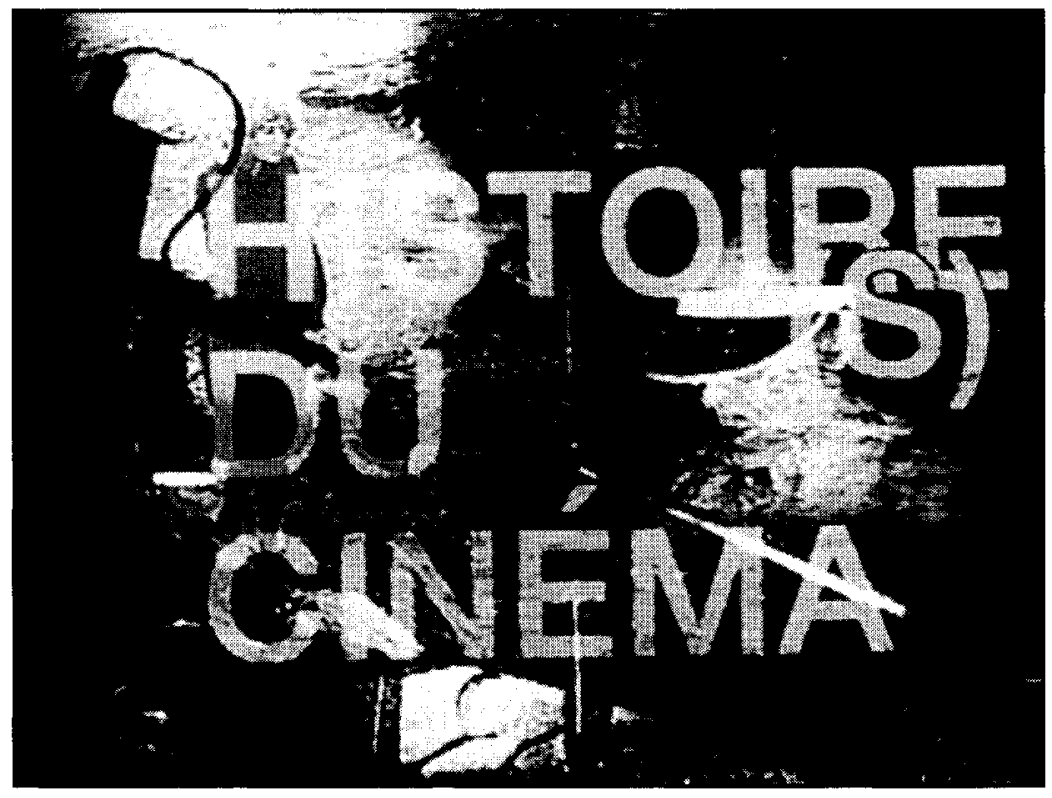

Histoire(s) du cinéma de Jean-Luc Godard (1997)

Collection Cinémathèque québécoise 
devait comprendre 10 épisodes en tout (sept sont réalisés à la date où j'écris), également groupés par paires. Aux premiers projets détaillés en ce sens ' a succédé, avec le passage du temps et le changement du désir, une suite plus informelle, dans laquelle frappe en outre l'absence de progression. Le matériau des épisodes 2A-2B (réalisés en 1993-1994), 3A-3B (1995-1996), 4A (1996-1997), est en grande partie le même que celui du couple 1A-1B (1988-1989); des pans entiers des épisodes les plus récents ont été enregistrés des années auparavant; outre le fragment d'entretien Godard-Daney, c'est le cas de ceux qui montrent des acteurs lisant ou récitant des textes (Julie Delpy, Sabine Azéma, Alain Cuny — datant, pour autant qu'on puisse juger, de 1986 — sans oublier Godard lui-même, qui joue beaucoup dans ses films ${ }^{2}$ ). Le système, sa structure et sa typologie se brouillent à mesure que chaque épisode ressasse davantage. Pourtant, la série avance, le programme se réalise, l'histoire se fait.

L'histoire que pratique Jean-Luc Godard est d'un type facilement identifiable: c'est l'histoire philosophique, celle que Hegel distingue, dans l'Introduction des leģons sur la philosophie de l'histoire, de l'histoire originale et de l'histoire réflexive, comme se distingue l'intérêt pour la pensée de l'intérêt pour le présent ou pour l'histoire. Hégélienne, l'Histoire des Histoire(s) du cinéma est cependant une histoire philosophique qui aurait perdu sa Raison; si l'histoire universelle dont parle Godard dans l'histoire du cinéma est rationnelle, sa raison est le chaos, la perte, l'éloignement de l'Esprit. C'est une histoire écrite par quelqu'un qui a eu 20 ans à l'époque de l'absurde (le Mythe de Sisyphe est de 1942, mais a été lu après la guerre). Pour rester chez Hegel, on pourrait la qualifier comme une histoire qui se déroulerait dans le temps de la culture, ce temps où l'esprit est devenu "étranger à soi-même", et que le philosophe oppose au temps de l'esprit vrai, où la conscience est dans une unité immédiate avec son essence (Hegel, p. 50).

Jean-Luc Godard, sans doute, oppose art et culture; c'est même l'un des thèmes favoris de ses entretiens ${ }^{3}$. Il ne peut cependant ignorer que le cinéma est, ou a été, partie prenante du développement de l'industrie culturelle, et à ce titre, a pu apparaître comme directement opposé à l'art, ainsi que l'ont 
souligné, entre autres, les tenants de l'école de Francfort ${ }^{4}$. L'histoire du cinéma apparaît donc, à l'historien philosophe - celui qui cherche la raison de l'histoire et la Raison dans l'histoire comme l'histoire d'une perte de l'Art, mais d'une perte paradoxale, puisqu'elle se décrit aussi comme l'histoire d'une "fin " à l'art. Le cinéma est dans l'art; il a suscité des artistes sui generis, héritiers des artistes historiques de la peinture ou de la musique. En même temps, il est d'entrée de jeu le lieu d'une perte; aussi bien, à l'intérieur même du cinéma, on ne cesse de perdre ce qui a existé, plainte godardienne déjà ancienne (elle commence avec Tout va bien en 1972): on ne sait plus faire Eisenstein, on ne sait plus faire Murnau, ni même leur bâtard, Hitchcock...

Dans le mouvement, dialectique à coup sûr, de la réflexion de Godard, comme dans le mouvement de la pensée d'Adorno, se retrouve la même nostalgie d'un idéal passé: chez l'un et chez l'autre, ce qui est perdu est toujours présenté comme un état d'équilibre et d'harmonie, comme une organisation et une composition, bref, comme un idéal classique. Mais si Godard implicitement partage le credo, cher à Éric Rohmer, d'un apogée classique de l'art, Horkheimer et Adorno ont une perspective un peu différente: la façon dont l'art s'est d'abord écarté du classicisme était intéressante, et le détail ou l'effet y étaient devenus "[...] le véhicule de la révolte contre l’organisation" (p. 134); au contraire, l'industrie culturelle ne connaît plus que les effets, et, entre l'ensemble et les parties, il n'y a ni opposition ni cohérence, parce qu'il n'y a plus œuvre, mais seulement formule.

La position du sujet artiste Godard est décalée par rapport à ce débat esthético-idéologique: il est quelqu'un qui a été peintre, qui aurait aimé être romancier à la NRF, mais qui devient cinéaste, parce que la peinture, la littérature, au fond, ont tout dit. Il devient artiste sans illusion excessive sur l'art. L'ironie adornienne vise peu ce sujet qui s'accepte artiste en sachant que, peut-être, l'art a été dévoré par la culture ${ }^{5}$ - et qui, un peu plus tard, se cherchera historien en pensant qu'on ne peut faire d'histoire que dans une situation de "fin de l'Histoire" (non sans, parfois, la traduire secrètement en un "après moi le Déluge ") :

Lhistoire du cinéma est intéressante à faire comme le dernier chapitre de l'histoire de l'art, qui est lui-même 
le dernier chapitre d'un certain type de civilisation indo-européenne. Les autres civilisations n'ont pas eu d'art. Non qu'il n'y ait pas eu des poteries en Chine, des romans au Japon, au Mexique, c'est pas ça, c'est que l'idée de l'art est européenne. Ce n'est pas par hasard qu'on parle de l'Europe aujourd'hui, c'est parce qu'elle va disparaître. (Godard, cité dans Daney, p. 26a).

L'histoire se fait depuis sa fin, mais cela donc veut dire deux choses, ou plutôt, a deux valeurs. D'abord, c'est depuis la fin que s'ordonnent les perspectives; dans l'insistance de Godard sur la fin de lhistoire, il eritre également cette préoccupation, foncièrement classique, des perspectives ordonnées; il faut pouvoir réfléchir l'œuvre ou l'idée en même temps qu'on l'invente, et c'est l'aspect positif de cette position apocalyptique. Mais en outre, cette histoire qui est celle d'une perte se fait depuis une position subjective fondée sur une psychologie de la perte et de la mort. Vers le milieu de l'épisode $2 \mathrm{~B}$, Godard cite le long monologue intérieur du major Amberson qui, sentant la mort prochaine, fait défiler sa vie mentalement, comme si la vie ne pouvait se ressaisir que depuis le point de vue de la mort; mais dans l'épisode $1 \mathrm{~A}$, c'est la "fin" du cinéma hollywoodien qui est convoquée, avec la longue

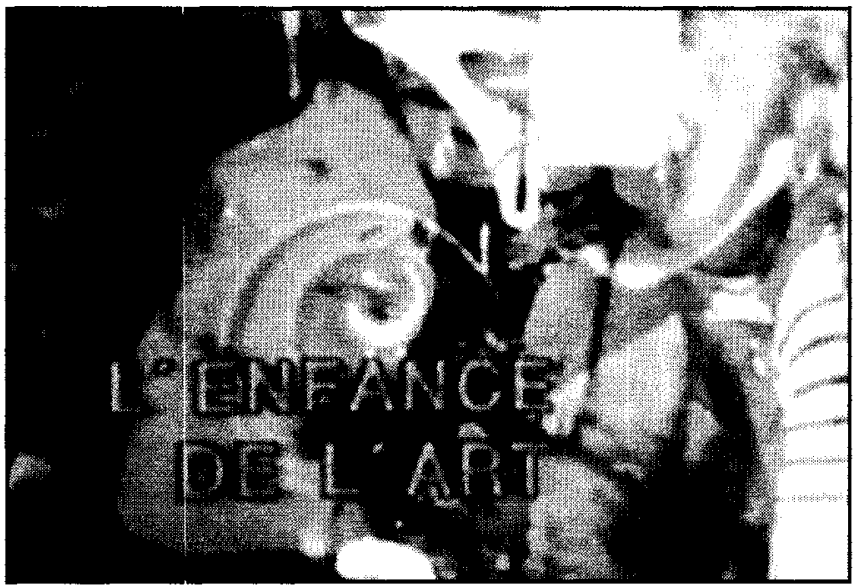

Histoire(s) du cinémo de Jean-Luc Godard (1997)

Collection Cinémathèque québécoise 
et dense réflexion à propos du retour du réel et de sa revanche sur l'usine à imaginaire et son règne artificiel. Au fil des épisodes, inextricablement, la perte est à la fois celle de l'enfance de l'artiste et celle de l'enfance de son art (un des cartons récurrents est justement: L'ENFANCE DE L'ART $^{6}$ ): à l'ère de la télévision, triste âge adulte de l'enfant ou de l'adolescent qui fut aimé sous le nom de "cinéma", se superpose le mûrissement doucement triste de l'artiste Godard, l'âge des désillusions, la perte, ordinaire mais irrémédiable, de la jeunesse et de ses espoirs.

Les films ne cessent de signifier cette confusion délibérée entre un double régime, artistique et subjectif, de fin et de perte, et c'est comme multiplement incarnée par Jean-Luc Godard que s'offre l'histoire $^{7}$ : par la constante énonciation en première personne (à l'image et au son); par le biais, plus discret mais aussi insistant, de jeux de signifiants tel celui-ci :

\section{HISTOIRE}

\section{TOI}

(le sujet comme reste chu de l'Histoire); et bien sûr, par la constante assertion, dans les monologues comme dans les citations, de la même thèse crépusculaire: le grand soleil du cinéma rougeoie peut-être encore, mais c'est qu'il est proche de se coucher; quant au cinéaste, il approche aussi de sa fin, et c'est de décembre, dernier mois de l'année, qu'il date son autoportrait.

\section{2}

A deux reprises, la seconde répétant presque littéralement la première - fin de l'épisode $1 \mathrm{~B}$, fin de l'épisode $2 \mathrm{~B}-$, Godard explique que, si la photographie a été inventée en noir et blanc, ce n'est pas à cause d'un manque technique; " [...] mais comme, en reproduisant la vie, on se préparait à lui retirer jusqu’à son identité, on porta le deuil de cette mise à mort, et c'est avec les couleurs du deuil, le noir et le blanc, que la photo se mit à exister». Un peu plus loin : "[...] pour masquer le deuil, les premiers Technicolor prendront les mêmes dominantes que les couronnes mortuaires. [...] Parce qu'il faut porter le deuil mais en l'oubliant, et madame de Staël nous a dit comment. Elle écrit à Napoléon: "La gloire, Sire, est le deuil éclatant du bonheur". " 
Deuil éclatant (les sunlights, les oscars, les festivals) du bonheur (les couleurs de la peinture, les couleurs de la vie, les couleurs de la beauté) -, mais le bonheur lui-même est-il si désirable? On peut en douter : à l'épisode $1 \mathrm{~A}$ est rapportée cette phrase de Max Ophuls: "Le bonheur n'est pas gai."

La perte, dans tout cela, est donc logiquement associée au deuil, à la tristesse, et indirectement à l'abattement, à la mélancolie (une mélancolie que JLG / JLG. Autoportrait de décembre (1995) avouera comme telle). Mélancolie? Mais qui est mélancolique? Est-ce l'artiste, est-ce le sujet Godard, est-ce son œuvre, ou sa pensée? Ou bien, ne serait-ce pas l'Histoire elle-même, ou l'époque, ou l'entreprise historique en général? La mélancolie est avérée, c'est son sujet qui fait défaut, ou au moins, qui est labile. D'ailleurs, il n'est pas dit que cette mélancolie soit à mettre sur le même plan que le deuil qui, tout aussi certainement, habite les Histoire(s). On se souvient que l'article de Freud qui établit le parallèle canonique du deuil et de la mélancolie pose que, face à la privation de l'objet d'amour, le premier est la réaction saine, faisant passer dans le symbolique la douleur de la perte, tandis que le mélancolique réagit à la perte réelle de l'objet par une régression narcissique qui fait apparaitre l'ambivalence de son amour (le mélancolique de Freud se fait du mal parce qu'il ne peut plus en faire à l'objet perdu).

Pris littéralement, ce diagnostic n'éclaire que très approximativement les Histoire(s) (et même un film avouant la tristesse, comme $J L G / J L G$ ). Sans doute, la plainte godardienne est celle d'un amoureux qu'empoigne la douleur d'une perte; sans doute, il a aimé le cinéma plus que tout puisque aussi bien, il confesse qu'il l'a aimé littéralement comme lui-même. Mais cette perte est-elle bien celle du cinéma? Le cinéma est-il vraiment perdu, perdu à jamais? N'est-ce pas, dans les Histoire(s), une partic seulement du cinéma, celle qui s'appela tantôt montage, tantôt mise en scène ${ }^{8}$, qui est perdue? Autrement dit, n'est-ce pas, très directement, la pratique personnelle du jeune Godard qui s'est perdue simplement parce qu'il a vieilli, et n'est-ce pas seulement sa propre vieillesse qu'il déplore?

Quittant ces banalités psychologiques (peu de gens se réjouissent de vieillir), ill faut donc plutôt se demander ce que pourrait 
être une "mélancolie historique": comment une œuvre poétique à propos de l'histoire d'un art peut-elle inclure une dimension ou une qualité mélancoliques? Autrement dit, il s'agirait de comprendre ce qu'est cette place de la Mélancolie qu'occupe, mué en auteur d'histoire(s), le poète Godard. L'hypothèse que je ferai est que cette place est dessinée moins par la clinique que par le mythe - ou, cela revient au même, que la Mélancolie est à comprendre comme attitude méditative plutôt que comme situation psychologique. A plusieurs reprises, dans ces films, comme déjà dans Soigne ta droite (Une place sur la terre), Godard cite d'importants et significatifs fragments du roman de Hermann Broch, La Mort de Virgile. On sait que Broch termina ce roman en exil, comme un testament littéraire et esthétique avant la lettre. Il y imagine les derniers jours du poète latin, en proie aux affres physiques de la maladie (une malaria qui devait l'emporter à 51 ans), et aux affres psychiques du doute: fallait-il laisser survivre une œuvre, l'Énéide, qui n'avait, au mieux, atteint qu'à la beauté, sans jamais toucher au réel? Non, car "[...] rien d'irréel n'a le droit de survivre" (Broch, p. 223) ": il faut donc détruire l'Énéide, et le roman est le récit de cette obsession du poète, détruire son œuvre avant de mourir, sans s'en laisser dissuader ni par les amis ni par l'ange que les dieux secrètement lui envoient.

Les quatre longs chapitres de La Mort de Virgile portent chacun un titre qui comporte le nom d'un des éléments, et aussi, qui organise l'avancée de ce voyage initiatique vers le pays originel : celui de la poésie, celui des ombres, l'au-delà d'où nous provenons et où la réminiscence nous fait incessamment retourner (le Virgile de Broch est éminemment platonicien). Sans doute, le romancier viennois, exilé en même temps et au même lieu que Freud, a pensé à la structure par cercles de L'Enfer de Dante, qui mettait en scène un autre Virgile: le guide, l'appui et le "référent". Godard a souvent dit que Broch fut l'un de ses écrivains de chevet (surtout Les Somnambules); citant cette œuvre crépusculaire, il ne peut manquer de provoquer un double parallèle: Virgile / Dante, Broch / Godard - donc aussi Virgile / Broch, Dante / Godard. Les Histoire(s) n'oublient pas l'auteur de la Divine Comédie, figuré à l'épisode 4A sous les 
traits que lui prêta Uccello dans son Déluge; mais Godard avait de longue date repris et travaillé cette configuration du poète que guide un autre poète avec Le Mépris (1963), où la figure de Fritz Lang est une sorte de Virgile moderne, tandis que Dante (et Hölderlin, celui qui en un sens accomplit la promesse de poésie pour laquelle meurt Virgile) y était un ange tutélaire. Le poète mourant n'est-il pas, d'ailleurs, le superpoète, le poète par excellence? Et, de Proust (qui parodiant Jean-Baptiste disait de la Recherche: "Il faut qu'elle croisse, et que je diminue.") à Broch lui-même, qui meurt sur les dernières pages de son dernier livre, n'a-t-on pas là une allégorie de la situation de Godard, lorsqu'il poétise la ruine de son art, le cinéma?

Était-il, maintenant, désigné lui-même pour veiller? Jamais, au grand jamais, il n'en serait capable, lui qui était incapable de porter secours, rebelle à secourir les autres, lui, le faiseur de mots, qui était forcé d'anéantir son œuvre parce que l'humain, parce que l'activité humaine: et la détresse humaine avaient eu pour lui si peu d'importance qu'il n'avait rien pu en retenir avec amour dans ses peintures ou même dans ses fables, que tout était resté sans être noté, car il s'était contenté de le glorifier et de le transformer en beauté, inutilement (Broch, p. 210)!

Ces phrases, qui appartiennent à la conclusion de la longue méditation ou du long délire nocturne de Virgile, en proie à la fièvre et au regret, peut-être Godard se les est-il appliquées à luimême, à un défaut fondamental de son ouvre qui serait en effet de ne rien "retenir avec amour». Plus sûrement, il a dû y voir en filigrane le destirı du cinéma, la fatalité d'un art qui incessamment a risqué de manquer le réel, pour avoir trop cherché la beauté.

Tout homme, sans doute, pense obscurément que l'histoire n'a eu lieu que pour lui, n'a été écrite que pour lui, qu'elle n'est qu'un roman (ou un essai) écrit pour qu'il en tire la leçon ou le bénéfice. Mais il en est qui pensent que c'est le monde qui est un livre (ou "fait pour aboutir à un beau livre", comme Mallarmé). Le Godard des Histoire(s) du cinéma voudrait, aurait voulu prendre le monde et en faire un beau livre. Mais comment prendre le monde quand, ce sont ses propres termes, on 
est né à 20 ans, et dans un musée? Sans doute, un long passage de l'épisode 3B le rappelle, le cinéma n'est pas n'importe quel musée : c'est le musée du réel. Mais même dans "son " musée, le réel ne figure que par des œuvres qui se substituent à lui en le symbolisant ("c'était ça la politique des auteurs: pas les auteurs, les ouvres"), de même que les Histoire(s) du cinéma sont une méditation sur les rapports entre des cuvres, et non pas, directement, sur les rapports entre des événements réels.

Qu'est-ce donc que l'Histoire? Godard, qui ne rate pas une occasion de manier l'équivoque entre histoire et Histoire, n'oublie pas la préoccupation du récit (memoria rerum gestarum). Mais si l'Histoire, ce n'est que des histoires, il est aisé de ne lui accorder aucune importance: elle ne fait que se répéter, identique sous des oripeaux variés, "[...] le contenu essentiel est partout le même. Eadem sed aliter" affirmait Schopenhauer. Les mêmes choses, mais autrement: la formule s'applique indifféremment aux histoires, aux scénarios (ceux des films ou ceux de l'Histoire: les mêmes, toujours), et tout cela n'a pas la moindre signification générale. Godard, qui hérite comme Rohmer du pessimisme schopenhauerien, le reçoit cependant, peut-être inconsciemment, à travers Nietzsche, qui fut plus sensible à la nature du discours de l'Histoire. Un discours puissant, d'ailleurs, susceptible d'affecter la vie, parce que l'Histoire est à la fois pensée, don et munition. On retient souvent la condamnation nietzschéenne de l'histoire, laquelle, en accroissant la distance entre l'homme et son dehors, entre l'être et le temps, "trouble les instincts" et engendre l'ironie, le cynisme, l'épigonisme; sans doute, pour Nietzsche, le bonheur est dans l'oubli de l'Histoire; celle-ci néanmoins appartient au vivant, et au moins pour trois raisons: le vivant est actif et aspire (et c'est l'histoire monumentale); il conserve et vénère (histoire antiquaire); il souffre et a besoin de délivrance (histoire critique).

Conserver. Vénérer. Souffrir. Être délivré (ou consolé). C'est, très largement, aussi le programme proprement historique des Histoire(s) du cinéma. L'Histoire comme piété, l'Histoire comme réflexion: indéniablement, les deux traits majeurs de ce(s) film(s). Godard, à l'inverse de Schopenhauer, croit manifestement à la signification métaphysique de l'Histoire. L'histoire de 


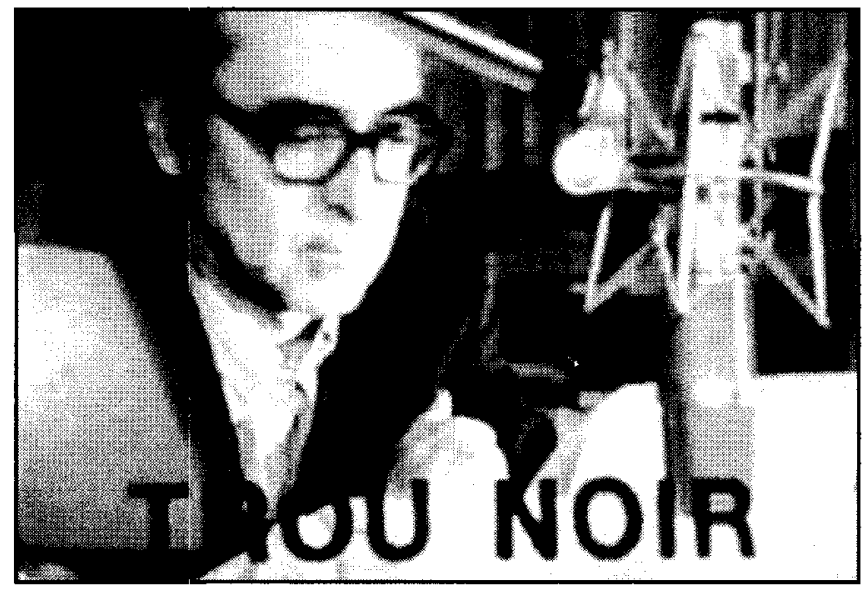

\section{Histoire(s) du cinéma de Jean-Luc Godard (1997)}

Collection Cinémathèque québécoise

l'art a en outre une vertu consolatrice, qui va de pair avec la litanie, la "théorie" des grandes figures (grands hommes - et femmes - grands films, grands emblèmes); dans l'épisode 3B, à la phrase du Christ de l'Évangile de Pasolini, "Voici la prière de chaque jour ", succède une liste de titres exemplaires, L'Espoir, La Poursuite infernale, L'Avventura. C'est, littéralement, l'écho dernier des idées hégeliennes reformulées par Malraux: l'art comme succédarié de religion, donnant accès à un "divin" sans Dieu $^{10}$. Souffrance et consolation; vénération et conservation: la mélancolie est ambiguë, à tout le moins, dans ce bon usage de l'histoire (comme Pascal parlant du bon usage des maladies). Il est donc temps de se souvenir que cette histoire est écrite, non par un historien, mais par un poète.

Le sens actuel du terme "mélancolie", celui qui l'associe à la tristesse et à l'abattement, s'est solidifié au début du XIX ${ }^{\mathrm{c}}$ siècle, chez Esquirol: "Ce n'est pas une douleur qui s'agite, qui se plaint, qui crie, qui pleure, c'est une douleur qui se tait, qui n'a pas de larmes, qui est impassible» (cité d'après Pigeaud, p. 66). Toutefois, dans le mouvement même où il proposait cette 
définition, Esquirol proposait aussi d'en finir avec le mot de mélancolie, qu'il trouvait ambigu, trop chargé, trop littéraire.

Le mot, il est bien vrai, a une longue histoire. La notion de mélancolie (étymologiquement "humeur noire" ou "bile noire») s'élabore durant l'Antiquité à partir de trois sources et dans trois directions ":

- c'est Hippocrate d'abord qui en fait une maladie (une folie), sans fièvre, liée à la tristesse et au mal-être (la dysthymie); Lucrèce rapportera ce malaise existentiel à la conscience de la mort et à la peur qu'elle engendre ${ }^{12}$;

- le pseudo-Hippocrate des Lettres donne du mélancolique le portrait, aujourd'hui accoutumé, du solitaire qui recherche les lieux déserts : la mélancolie fait rechercher la solitude, mais moins sous la forme de la retraite du Sage que sous la forme de la misanthropie;

- enfin, dans le Problème $X X X$ attribué à Aristote apparaît l'association de la mélancolie et du génie. La première phrase de ce texte célèbre en donne l'essentiel in nuce: "Pour quelle raison tous ceux qui ont été des hommes d'exception en ce qui regarde la philosophie, la science de l'État, la poésie ou les arts, sont-ils manifestement mélancoliques?» La mélancolie ici va de pair avec la capacité à l'inspiration, à la possession par les Muses ou par une autre force divine, à l'extasis ou furor (poétiques).

C'est cette triple définition et cette triple dimension, souvent ramenées à une dialectique entre furor et dysthymia, qui fut retravaillée par les théoriciens de la Renaissance, notamment Marsile Ficin, lequel donna sa forme glorieuse et canonique à l'image du génie mélancolique, en proie à l'exaltation et alternativement ou simultanément à l'abattement, au furor divinus comme à la déprime ${ }^{13}$.

Sans aucun doute aussi, c'est de cette image, complexe et historiquement stratifiée, du mélancolique qu’il faut se souvenir pour parler de Jean-Luc Godard et de ses Histoire(s) du cinéma. Chez Ficin, la dialectique de l'exaltation et de la dépression est hiérarchisée: le saturnien (l'artiste) est mélancolique; le salut lui vient du furor divinus, de l'enthousiasme qui l'arrache à l'acedia (que Ficin conçoit et décrit de façon proche de ce que nous 
appelons l'angoisse); donc, la mélancolie est la condicion de la vision, mais la vision excède cette condition première. De même, la force du texte pseudo-aristotélicien rangé dans les Problèmes au numéro XXX est de lier la poésie à l'inspiration, mais en suggérant que cet Autre qui inspire le poète (la Muse, la force divine) est en nous. Jackie Pigeaud, qui relève ce trait, observe en outre que, la poésie étant conçue comme force d'autant plus grande qu'on la fait opérer de plus loin, le mélancolique est enclin à la métaphore, à l'établissement de rapports et de semblances qui, sans lui, demeureraient cachés.

A ce dernier trait, il est impossible de ne pas voir à quel point cette figure du mélancolique saturnien, en proie à l'exaltation et à la dépression, inspiré et abattu, convient au cinéaste Godard ${ }^{14}$. Les Histoire(s) cultivent la métaphore, la sèment, la font grandir, la récoltent, l'égrènent; ou peut-être faudrait-il dire que la métaphore en est l'humus. On peut, à vrai dire, prendre la série de ces films à peu près n'importe où, et de là suivre le fil des associations, des chaînes signifiantes, des transports de sens, des migrations et des miroitements, bref, le travail du métaphorique. Vers le milieu de l'épisode $1 \mathrm{~B}$, les deux frères Lumière servent d'abord à activer ou à réactiver l'une des chaînes et des configurations les plus récurrentes de l'ensemble, celle de la lumière (le dispositif cinématographique tel que décrit par Godard est une lumière qui vient trouer l'ombre, il est cette lumière qui surprend les ténèbres dans leur dos, et qui se projette sur un écran, permettant à partir de là de passer à l'idée de la projection et à toutes les images qu'elle convoque). "Ils auraient pu s'appeler Abat-jour, mais ils se sont appelés Lumière": par cette plaisanterie de potache, Godard souligne la valeur quasi miraculeuse de ce patronyme (il n'est pas le premier à remarquer l'admirable de la coïncidence); mais le signifiant "abat-jour" ne reste pas lui-même inemployé, et trouve son prolongement dans l'espèce de visière transparente de joueur de tennis qu'arbore Godard à l'épisode $2 \mathrm{~B}$, ce que, justement en français balzacien, on appelait un abat-jour ${ }^{15}$. Dans le même passage sur les Lumière, du fait qu'ils "avaient la même bobine " se déduit qu'ils étaient faits pour inventer le cinéma, affaire de bobines allant par deux ${ }^{16}$; la suite continue à filer la métaphore des bobines, ou plutôt, la 
transforme, en changeant subrepticement le thème tout en gardant le phore: des images de bobines de film sur support nitrate décomposé, attestant de la périssabilité de la pellicule, traduisent ou illustrent la célèbre prédiction sur l'"invention sans avenir", tout en en distordant subtilement le sens...

Très souvent, Godard a insisté sur le fait que, pour avoir inventé le cinéma, il avait fallu être deux: deux frères Lumière, comme il y a deux bobines sur le projecteur ou la table de montage, mais aussi, comme la projection répond à l'enregistrement, le projecteur à la caméra. Le passage que je viens d'évoquer mêle cette idée du double ou du dédoublement à l'idée même de la décomposition, de la tristesse, de la perte (nitrate pourri, invention sans avenir). On trouve exactement le même mécanisme, pour ne citer qu'un autre exemple, dans la longue et brillante réflexion de l'épisode $1 \mathrm{~A}$ sur les hommes d'action et de pouvoir: à un éloge (sans doute sincère, dans ce contexte et ce momentlà, du moins) d'Irving Thalberg succèdent d'autres éloges adressés à des figures héroïques, celle de Charles Lindbergh, celle de Charles Foster Kane, mais insidieusement, on passe alors au portrait d'un autre magnat du film, impuissant, vicieux, évidemment mélancolique, Howard Hughes (à qui l'on ne peut pas ne pas penser que Godard fugitivement s'identifie un peu), qui amène comme naturellement une des nombreuses litanies des Histoire(s), celle des "films jamais faits": La Condition humaine, Don Quichotte, Humiliés et Offensés (comme par hasard, de grands titres de la littérature mondiale, qui entrent en même temps en résonance avec les titres de romans que Godard égrène au long de l'épisode, sur la bande-son) — tandis que commence une brève citation de la sonate "des Adieux" de Beethoven ${ }^{17}$ et qu'une image du Pré de Béjine ajoute, à la tristesse des films "jamais faits", l'horreur des films détruits.

Les deux exemples que je viens de décrire se fondent à peu près sur les mêmes ressorts: une correspondance directe entre des figures de grands hommes et des valeurs qui, par convention métaphorique, leur sont attachées. Il est une autre procédure sémantique, plus directe, plus crue, mais d'essence également métaphorique, reposant sur le jeu de mots et sa dissimulation (métaphore type du mélancolique). À l'épisode 1B, une 
réflexion, monologuée par la voix de Godard, sur le cinéma qui hérite de la photographie, "de ses droits et de ses devoirs", se superpose, à partir d'un certain point, à un fragment de la bande-son de Touchez pas au grisbi, de manière énigmatique (quel rapport entre Émile Zola, la photographie, Becker, Gabin, le grisbi ?). Or, le rapport, cryptique, est établi par un pur glissement du signifiant: de "nous héritons" on passe à "nous et Riton", le passage du Grisbi reproduit étant le long monologue où Gabin peste contre "ce con de Riton" qui les compromet et pourrait bien les perdre. Mais si l'opération est métaphorique, c'est bien sûr qu'on n'en reste pas à cette plaisanterie un peu gamine: le Grisbi, aussi bien, reviendra, à l'épisode 3B et à l'image cette fois, pour répondre à l'invective du Christ pasolinien, "Vous ne pouvez servir Dieu et l'argent", par une fusillade (le grisbi, faut-il le rappeler à qui aurait oublié l'argot des années trente, c'est l'argent). De ces allusions, jeux de mots semicachés, voire cryptés, les films sont pleins: c'est l'un des exercices intellectuels et poétiques où Godard excelle, et que visiblement il aime.

A la fin d'un long passage, à l'épisode $3 \mathrm{~A}$, sur la collaboration dans le cinéma français (qui reprend l'argument d'un passage semblable de l'épisode 1A), il est plusieurs fois question d'un train de 1942 qui mena en Allemagne des acteurs du cinéma français: tandis que la voix de Godard termine ce passage par cette phrase: "Hélas, oui, hélas, j'étais seul aussi à penser qu'ils étaient plusieurs encore dans ce train de 1942" s'inscrit le texte suivant sur les images: dans le train d'après il y avait / cette conne d'Irène / son train partait pour Auschwitz. La "conne" en question figure sur la couverture d'un livre, mais l'inscription ne peut pas, non plus, ne pas évoquer le célèbre roman érotique d'Aragon (publié anonymement), Le Con d'Irène. Juste après, commence la lecture d'un poème d'Aragon, par une jeune actrice à qui Alain Cuny viendra gentiment demander, du fond d'un jardin: "Eh! bien, ma chère Marie, ce train, est-ce qu'on le prend ou est-ce qu'on ne le prend pas?» Dans ce tressage inlassable des signifiants, au cœur d'un petit réseau de signifiés, se love le jeu du mélancolique avec la métaphore. 


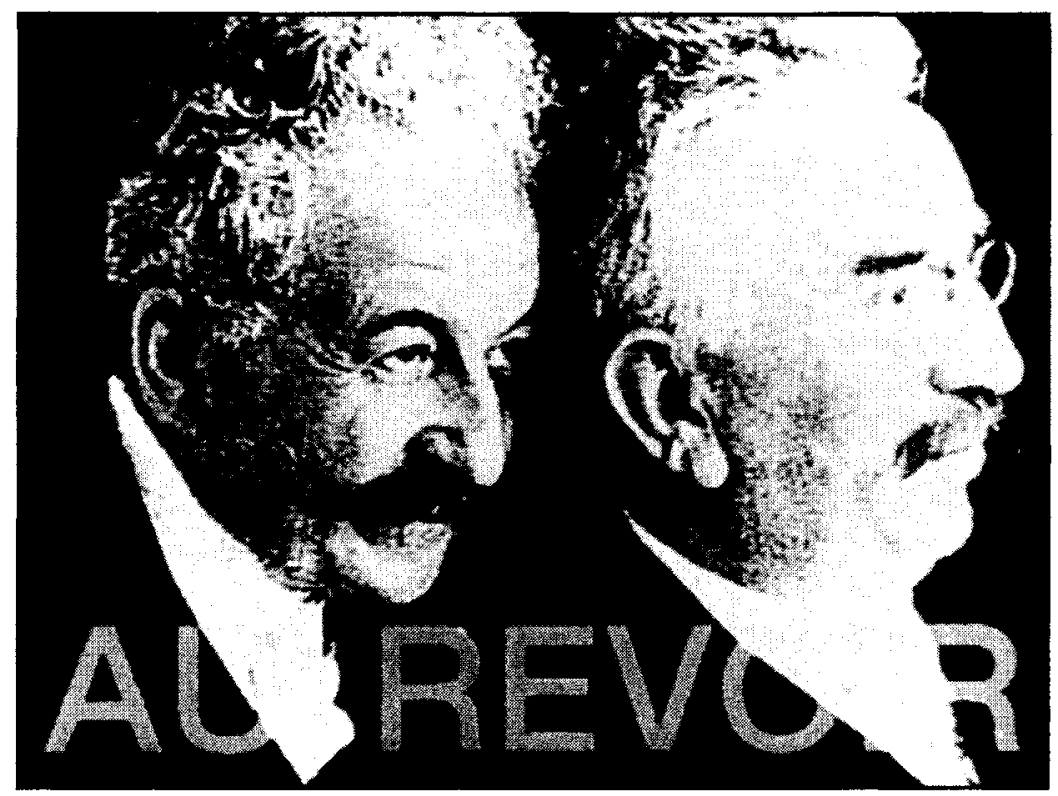

\section{Hisfoire(s) du cinémo de Jean-Luc Godard (1997)}

Collection Cinémathèque québécoise

[...] il nous faut nous reporter à La Mort de Virgile où, comme on le sait, l'Énéide doit être brûlée dans l'intérêt de la connaissance et où cette vérité est sacrifiée ensuite à l'exigence amicale de l'empereur derrière laquelle prédominent les impératifs très politiques er très orientés vers la pratique de l'époque (Arendt, p. 146).

Dans le roman de Broch, le poète meurt, mais son œuvre au contraire entre dans l'éternité (l'éternité relative des œuvres humaines), au prix d'une sorte de malentendu: le défaut primordial qu'y percevait l'auteur n'est, littéralement, rien au regard de son utilité politique, pragmatique, mondaine.

Sans doute il ne faut pas tout transposer, même si le livre de Broch, que Godard cite continûment depuis plus de 10 ans (probablement depuis qu'il l'a $l^{18}{ }^{18}$ ), répond manifestement à certaines de ses préoccupations intellectuelles et esthétiques inti- 
mes. L'idée d'éternité, notamment, ne saurait avoir le même sens pour l'empereur romain qui décide de préserver un monument poétique érigé à son peuple et à sa civilisation, et pour le cinéaste de Pierrot le Fou (1965), qui, venant après Rimbaud, pouvait en reprendre la fameuse définition ( $C$ ' 'est la mer mêlée au soleil»), laquelle fait échapper l'éternel à toute affaire humaine. Néanmoins, dans ce profond et brûlant conflit entre deux sortes (on voudrait presque dire deux races) d'intérêts, la politique et la poétique, on sent bien que Godard a trouvé un écho à son souci envers l'histoire de l'art, envers l'histoire du cinéma. Faut-il croire que le cinéma tout entier est sénile, va mourir, est déjà mort (c'est-à-dire: faut-il le vouloir) ? Faut-il au contraire y chercher ce qui, dernière incarnation, même dégradée ${ }^{19}$, de l'Esprit, va en préserver la dernière forme possible?

Pour un historien, de l'espèce assez spéciale qui est celle de Godard, c'est-à-dire un historien capable de dire, comme Flaubert de Madame Bovary, que l'histoire "c'est lui", la question évidemment se déplie aussitôt. "Faut-il continuer à croire au cinéma?" devient: "Faut-il continuer à faire des films?" Et quels films? Hantés par l'autosuggestion de l'impuissance à raconter des histoires, les derniers longs métrages du maître du lac Léman sont en fait de très belles, très denses, très romanesques et profondes histoires: histoires d'identité (de Soigne ta droite à Hélas pour moi, 1994), histoires d'hommes et de femmes (de Nouvelle Vague, 1990, à Forever Mozart, 1997), histoires de représentation (tous). Les Histoire(s) du cinéma ont été les entractes de ces grandes et pessimistes fictions, comme si le passage par l'histoire de l'art était nécessaire, malgré le discours sur la perte, sur la fin et sur la vieillesse de l'Art, à recharger d'enthousiasme, à exalter de nouveau l'artiste, le cinéaste et le conteur. L'Histoire du cinéma y devient à son tour une sorte de figure mélancolique et géniale, "transmutant ${ }^{20}$ " la tristesse et la fatigue en exaltation et en enthousiasme.

Les grands noms de l'Histoire sont toujours autant de Nomsdu-Père que l'historien, surtout l'artiste historien de son art, envisage comme ceux de ses pères à lui. La mort des pères, même s'ils sont plus jeunes que vous, comme dans le cas de Godard, l'ont été Truffaut voire Daney, a cette conséquence 
radicale et difficile qu'il faut bien accepter à son tour de prendre leur place, accepter le sacrifice d'être ou de devenir un père (parfois, pour ceux-là mêmes qu'on ne connaîtra jamais). Le dernier épisode réalisé des Histoire(s), le 4A, intitulé "Le contrôle de l'univers", est en partie consacré à Hitchcock, l'un des pères symboliques les plus manifestes de cette Nouvelle Vague qui, pour Godard, est devenue l'équivalent général du cinéma d'art. Père terrible, père puissant, réussissant ce qu'aucun de ses enfants jamais ne saura réussir : la maîtrise ( je suis maître de moi comme de l'univers"); Hitchcock fut le maître des affects, et de lui ne resteront certes pas les histoires, insignifiantes par ellesmêmes, qu'il a racontées, mais les signifiants-maîtres par lesquels il les a utilisées pour exercer sa terreur sur son esclave, le spectateur $^{21}$ : Godard relève, avec une admiration non feinte, communiquée sur un ton proche de l'extase, la liste de ces objets hitchcockiens (bouteille de pommard, moulin dont les ailes tournent à l'envers, avion saupoudrant des récoltes inexistantes, clé tombée dans une bouche d'égoût) par quoi la force fascinante de l'image a su trouver son acmé et sa justification.

L'image, après Hitchcock, a faibli, parce que l'univers a confié son contrôle à des sociétés anonymes de l'image, ces réseaux de télévision dont les sigles, régulièrement, ponctuent les Histoire(s) comme autant de désirs d'exorcisme, qui jouent sur la perversion et non plus sur la maîtrise. Si l'histoire doit rester la grande consolatrice, et non seulement par la force de pensée que le philosophe lui accorde et lui attache, il faut qu'elle soit aussi l'histoire d'un espoir. Le trait le moins étonnant de ces films n'est pas d'y voir revenir, avec autant d'exactitude et de régularité, une référence évangélique, et non pas tant celle, deux fois répétée, à l'évangile de Matthieu dans la version catholique de Pasolini, que la citation des deux grands saints du protestantisme ${ }^{22}$, Jean et Paul. On sait de reste comment ces deux prénoms ont circulé dans tout l'œuvre de Godard, depuis le Paul de Vivre sa vie (1962) jusqu'au Paul Godard de Sauve qui peut (la vie) (1979), depuis l'oncle Jean de Prénom Carmen (1983) jusqu'aux nombreux Jean (Vigo, Cocteau, Epstein, Renoir) dont Godard dans les Histoire(s) se fait l'homonyme ou l'éponyme. C'est à saint Paul, ou plutôt à l'un de ses commentateurs ${ }^{23}$, qu'il reprend une 
formule qui s'inscrit, elle aussi, à plusieurs endroits des Histoire(s): l'image viendra au temps de la résurrection.

Il y aura encore des images, il y aura encore de l'image: promesse d'un paradis futur (les Histoire(s) nous ont beaucoup emmenés dans clivers enfers). Les images, à vrai dire, n'en finissent pas de se succéder et l'historien n'en aura jamais fini avec elles comme le suggère, optimiste ou foncièrement pessimiste, une autre inscription, probablement tirée d'Ovide, à la fin de l'épisode 3A: "nam fit ut in somnis facere hoc videatur imago quippe ubi prima perit alioque est altera nata inde statu prior hic gestum mutasse videtur ${ }^{24}$." L'image qui vient la dernière semble changer toutes celles qui l'ont précédée: mais, ajoute-t-on, cela se passe dans les songes: autant dire, au cinéma.

\section{Université de Paris III-Sorbonne Nouvelle}

\section{NOTES}

1 Un des premiers états aboutis en est exposé par Jean-Luc Godard dans un entretien filmé avec Serge Daney ("Entretien avec Jean-Luc Godard", Libération, 26 décembre 1988), dont une partie est utilisée dans l'épisode $2 A$ des Histoire(s). Une autre transcription de cet entretien a été proposée dans un numéro récent des Cahiers $d u$ cinéma ( $\mathrm{n}^{\circ} 513,1997$, p. 49-55), mais elle est à l'évidence infiniment moins fidèle à l'esprit et au style godardiens que ne l'était la transcription de 1988, due à Daney luimême; je continue donc de renvoyer à cette version originale.

2 Julie Delpy a tourné dans Mauvais Sang, de Léos Carax (1986), qu'elle accompagnait au tournage de King Lear (1987) sur les bords du Léman; Sabine Azéma ressemble exactement à son personnage de Mélo (Alain Resnais, 1986); Alain Cuny avait joué dans Détective (1985). Il faut ajouter la lecture, par Maria Casarès, dont on reconnaîtrait la voix entre mille, d'un texte de Heidegger, sur la bande son de l'épisode $1 \mathrm{~B}$ : enregistrement probablement ad hoc pour le film de Godard, et dont peutêtre un épisode à venir nous offrira l'image.

3 Encore, le 18 mai 1997, au festival de Cannes, lors d'une conversation de près de deux heures avec un journaliste de télévision, Paul Amar (diffusée peu après sur la chaîne Paris-Première).

4 Exemple: "La différence entre la série Chrysler et la série General Motors est au fond une pure illusion qui frappe même l'enfant amateur de modèles variés. [...] Il en est de même pour les productions des Warner Brothers et de la Metro Goldwyn Mayer. Même les différences entre les modèles plus ou moins chers produits par la même firme s'amenuisent de plus en plus : pour les automobiles, elles se réduisent au nombre de cylindres, à la capacité, au degré de nouveauté des gadgets; pour les films, au nombre de stars, à l'étalage de technique, de travail, d'équipement et à l'utilisation des clichés psychologiques les plus récents" (Max Horkheimer et Theodor Adorno, "La production industrielle de biens culturels", Dialectique de la Raison. Fragments philosophiques (1944), Paris: Gallimard, 1974, p. 132-133). 
5 "Notre querelle avec Hollywood a toujours été: dès que vous faites du commerce, c'est autre chose, c'est de la culture" (Serge Daney, "Entretien avec Jean-Luc Godard ", Libération, 26 décembre 1988, p. 26a).

6 C'est aussi le titre d'un court métrage de Godard, réalisé en 1989.

7 "Je raconte mon histoire, avec le sentiment qu'elle n'est pas vraiment à moi, mais qu'il n'y a que par moi que je la connais. Elle est très simple, elle passe par le cinéma. » Cette phrase est tirée d'un entretien entre Noël Simsolo et Jean-Luc Godard radiodiffusé à France-Culture en 1989.

8 Faut-il rappeler que, pour lui, cela est ou a été la même chose. Voir notamment son texte intitulé "Montage, mon beau souci", initialement publié dans les Cahiers du cinéma en 1956 et réédité dans l'ouvrage Jean-Luc Godard par Jean-Luc Godard (Paris: Pierre Belfont, 1968, p. 52-55).

9 Cette phrase n'est pas reprise par Godard.

10 Au moment d'en venir à la clé de voûte de son édifice, la religion révélée, Hegel, dans la Phénoménologie de l'esprit, pose l'historicité des produits artistiques comme cadeau du destin, supérieur même à leur être éthique premier (Paris: Aubier, p. 261).

11 J'ai largement puisé, ici, dans les travaux de Jackie Pigeaud.

12 "Souvent même la peur de la mort inspire aux humains un tel dégoût de la vie et de la lumière qu'ils vont dans leur désespoir jusqu’à s'assurer de leurs mains le trépas, sans se souvenir que la source de cette souffrance était cette peur elle-même..." (Lucrèce, De la nature, Paris: Garnier, 1964, livre III, v. 79-80).

13 Sur cette conception Renaissance de la mélancolie, voir le locus classicus de Klibansky, Panofsky et Fritz Saxl, Saturne et la mélancolie (Paris: Gallimard, 1989). Sur Ficin, voir le livre d'André Chastel, Marsile Ficin et l'art (Genève: Droz, 1996).

14 Est-il nécessaire de souligner que, dans tout mon commentaire, je vise moins le sujet Jean-Luc Godard, dont $j$ 'ignore à peu près tout, que la persona construite dans les films et leurs entours? Comme il est dit (et écrit) à plusieurs reprises dans les Histoire(s): "L'histoire, pas celui qui la raconte."

15 Au début du Père Goriot, par exemple, mademoiselle Michonneau, l'une des figures de la pension Vauquer, est toujours décrite comme portant un tel "abat-jour".

16 Le numérotage des Histoire(s) lui-même en atteste: 1A-1B, 2A-2B, etc., est le numérotage traditionnel des bobines de films.

17 Sonate pour piano n" 26 en mi bémol, op. 81a (1809), également connue sous l'appellation Sonate caractéristique. Le même passage de cette même œuvre est cité par Godard dans $J L G / J L G$ et dans Puissance de la parole; il s'agit du début du deuxième mouvement, andante espressivo, qui, dans le programme de l'œuvre, est sous-titré "L'absence", - début qui consiste en une phrase éminemment interrogative et mélancolique.

18 La traduction française est parue en 1955, mais son édition en collection "de poche" (la collection "Limaginaire" de Gallimard) ne remonte qu'à 1980. Les premières citations, sauf erreur de ma part, interviennent chez Godard dans Soigne ta droite, réalisé en 1986; or, il est rare que Godard attende pour citer ce qu'il lit, ainsi qu'il l'avait d'ailleurs théorisé dans son article "On doit tout mettre dans un film" (Jean-Luc Godard par Jean-Luc Godard, Paris: Pierre Belfont, 1968, p. 391-393).

19 Lidée de la "dégradation des valeurs" est au cœur de tout l'œuvre de Broch, et notamment des Somnambules, dont c'est le sous-titre.

20 Au sens, on s'en doute, de l'Umwertung nietzschéenne.

21 "Avec Psycho, j'ai fait de la direction de spectateur", confiait-il lucidement à François Truffaut. 
22 "Tout cela te permet à présent de bien juger tous les livres et de distinguer lesquels sont les meilleurs. Ce sont nommément l'Évangile de Jean et les Épîtres de saint Paul [...] ainsi que la première Épître de saint Pierre qui forment le véritable noyau et la moelle de tout [le Nouveau Testament], ce pour quoi ils devraient à bon droit être les premiers." (Martin Luther, "Préface au Nouveau Testament", De la liberté du chrétien. Préfaces à la Bible, Paris: Seuil, 1996, p. 81).

23 Lidée se trouve dans la première Épitre aux Corinthiens: "Et tout comme nous avons porté l'image terrestre, nous porterons aussi l'image céleste" (XV, 49), mais la formulation citée par Godard, plus radicale, est d'un commentaire de cette épître par l'un des Pères de l'Église, Grégoire de Nysse.

24 Je n'ai pas retrouvé le texte dont provient ce fragment, et risquerai cette traduction grossière: "Car il arrive que, dans les songes, on voit une image faire cela, car, où la première est partie et est ailleurs, une autre, née de là, dans une position supérieure, semble alors avoir changé ce qui a eu lieu.»

\section{OUVRAGES CITÉS}

Arendt, Hannah. "Hermann Broch", Vies politiques. Paris: Gallimard, 1974.

Aristote. Problème XXX. Paris: Rivages, 1988.

Broch, Hermann. La Mort de Virgile. Paris: Gallimard, 1980.

Camus, Albert. Le Mythe de Sisyphe. Paris: Gallimard, 1942.

Chastel, André. Marsile Ficin et l'art. Genève: Droz, 1996.

Daney, Serge. «Entretien avec Jean-Luc Godard». Libération, 26 décembre 1988.

Freud, Sigmund. "Deuil et mélancolie (1917)", Euures complìtes, tome XII. Paris: P.U.F. (1988), p. 259-278.

Godard, Jean-Luc. "Montage, mon beau souci", Jean-Luc Godard par Jean-Luc Godard. Paris: Pierre Belfont, 1968, p. 52-55.

Godard, Jean-Luc. "On doit tout mettre dans un film", Jean-Luc Godard par JeanLuc Godard. Paris: Pierre Belfont, 1968, p. 391-393.

Godard, Jean-Luc. Introduction à une véritable histoire du cinéma. Paris: Albatros, 1980.

Hegel, G.W.F. Phénoménologie de l'esprit. Paris: Aubier, 1941.

Hegel, G.W.F. Leçons sur la philosophie de l'histoire. Paris: Vrin, 1967.

Horkheimer, Max et Theodor W. Adorno. "La production industrielle de biens culturels", Dialectique de la Raison. Fragments philosophiques. Paris: Gallimard, 1974.

Klibansky, Panofsky er. Fritz Saxl. Saturne et la mélancolie. Paris: Gallimard, 1989.

Lucrèce. De la nature. Paris: Garnier, 1964.

Luther, Martin. "Préface au Nouveau Testament", De la liberté du chrétien. Préfaces à la Bible. Paris: Seuil, 1996.

Nietzsche, Friedrich. « De l'utilité et de l'inconvénient de l'histoire pour la vie", Considérations inactuelles. Paris: Aubier, 1964.

Pigeaud, Jackie. Les Maladies de l'àme. Paris: Les Belles Lettres, 1986.

Pigeaud, Jackie. "Présentation", dans Aristote, Probleme XXX. Paris: Rivages, 1988. 